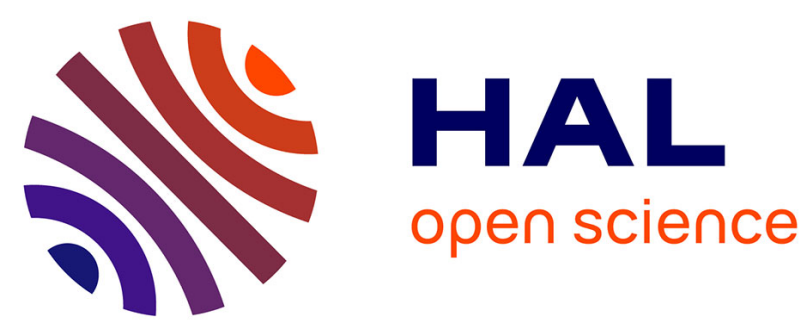

\title{
INTERFERENCE ANALYSIS IN RECONFIGURABLE INTELLIGENT SURFACE-ASSISTED MULTIPLE-INPUT MULTIPLE-OUTPUT SYSTEMS
}

Jiang Liu, Xuewen Qian, Marco Di Renzo

\section{- To cite this version:}

Jiang Liu, Xuewen Qian, Marco Di Renzo. INTERFERENCE ANALYSIS IN RECONFIGURABLE INTELLIGENT SURFACE-ASSISTED MULTIPLE-INPUT MULTIPLE-OUTPUT SYSTEMS. IEEE International Conference on Communications, Jun 2021, Toronto, Canada. hal03373532

\author{
HAL Id: hal-03373532 \\ https://hal.science/hal-03373532
}

Submitted on 11 Oct 2021

HAL is a multi-disciplinary open access archive for the deposit and dissemination of scientific research documents, whether they are published or not. The documents may come from teaching and research institutions in France or abroad, or from public or private research centers.
L'archive ouverte pluridisciplinaire HAL, est destinée au dépôt et à la diffusion de documents scientifiques de niveau recherche, publiés ou non, émanant des établissements d'enseignement et de recherche français ou étrangers, des laboratoires publics ou privés. 


\title{
INTERFERENCE ANALYSIS IN RECONFIGURABLE INTELLIGENT SURFACE-ASSISTED MULTIPLE-INPUT MULTIPLE-OUTPUT SYSTEMS
}

\author{
Jiang Liu, Xuewen Qian, and Marco Di Renzo \\ Université Paris-Saclay, CNRS, CentraleSupélec \\ Laboratoire des Signaux et Systèmes, 3 Rue Joliot-Curie, 91192 Gif-sur-Yvette, France \\ email: marco.di-renzo@universite-paris-saclay.fr
}

\begin{abstract}
Reconfigurable intelligent surfaces (RISs) are regarded as an emerging technology for the next generation of wireless communications. In this paper, we consider a multiple-input multiple-output network where each base station serves a user equipment with the aid of an RIS equipped with $N$ reconfigurable elements. We characterize the interference at one user equipment that is caused by the signal emitted by its non-serving (interfering) RIS. By assuming Rayleigh fading channels, we study the corresponding interference-to-noiseratio (INR) under the assumption of large values of $N$, and we prove that the INR is the product of a Chi-Square random variable (RV) and an RV that is approximated with a Gamma distribution. In addition, we prove that the amount of fading of the INR is equal to one in the large $N$ regime.
\end{abstract}

Index Terms - Reconfigurable intelligent surfaces, multiantenna transmission, performance analysis.

\section{INTRODUCTION}

A reconfigurable intelligent surface (RIS) is an emerging technology for increasing the reliability of wireless communications at a reduced hardware cost and energy consumption [1], [2]. An RIS is capable of realizing passive beamforming by adding customized phase shifts to the incident waves, thus appropriately shaping the scattered waves towards specified locations [3], [4]. In contrast with traditional relays, an RIS does not need power amplifiers, introduces no additive noise, and no self-interference in full-duplex implementations [5].

Modeling and analyzing the performance of RIS-aided wireless systems is an open research issue. As outlined in a recent survey and tutorial paper [2], several authors have investigated the joint optimization of the beamforming vector at the transmitter, the matrix of phase shifts at the RIS, and the combining vector at the receiver. However, very few papers have tackled the evaluation of the performance of multiple-input multiple-output (MIMO) RIS-aided systems in fading channels [6]. In [6], the authors have recently characterized the distribution of the signal-to-noise-ratio (SNR) by using tools from random matrix theory and by capitalizing on the optimization framework introduced in [7]. The existing
Table 1: Main notation $(\mathrm{RV}=$ random variable $)$

\begin{tabular}{|c|c|}
\hline Symbol & Definition \\
\hline$\stackrel{d}{=} \sim$ & Equivalent in distribution, distributed as \\
\hline$N \geqq 1, N \geqq 1, N \gg 1$ & Equality, approximation and scaling law if $N \gg 1$ \\
\hline $\begin{array}{l}\operatorname{diag}(\mathbf{x}) \\
\arg (x)\end{array}$ & $\begin{array}{l}\text { a diagonal matrix with diagonal given by vector } \mathbf{x} \\
\text { the angle of the complex variable } x \text {. }\end{array}$ \\
\hline $\mathbb{E}, \mathbb{V}$ & Expectation, variance \\
\hline$\Re, \Im$ & Real part, imaginary part \\
\hline$(\cdot)^{H},(\cdot)^{*}$ & Hermitian operator, complex conjugate operator \\
\hline$|\cdot|,\|\cdot\|$ & Absolute value, norm of a vector \\
\hline $\mathbf{0}_{A \times B}$ & $A \times B$ matrix with all zero entries \\
\hline $\mathbf{1}_{A \times B}$ & $A \times B$ matrix with all one entries \\
\hline $\mathcal{N}\left(m, \sigma^{2}\right)$ & Gaussian RV $\left(\mathbb{E}=m, \mathbb{V}=\sigma^{2}\right)$ \\
\hline $\mathcal{C N}\left(m, \sigma^{2}\right)$ & Complex Gaussian RV $\left(\mathbb{E}=m, \mathbb{V}=\sigma^{2}\right)$ \\
\hline $\mathcal{G}(k, \theta)$ & $\operatorname{Gamma} \operatorname{RV}\left(\mathbb{E}=k \theta, \mathbb{V}=k \theta^{2}\right)$ \\
\hline $\mathcal{X}^{2}(k)$ & Central Chi-Square $\mathrm{RV}(\mathbb{E}=k, \mathbb{V}=2 k)$ \\
\hline
\end{tabular}

works, however, consider a single transmitter, a single RIS, and a single receiver. Thus, they ignore the interference that is generated by the presence of multiple RISs that are optimized for serving their respective transmitters and receivers.

Motivated by these considerations, we analyze a twouser interference channel in the presence of two RISs. Each MIMO transmitter communicates with its intended MIMO receiver with the aid of an RIS. Each RIS is equipped with $N$ reconfigurable elements that are optimized to maximize the received SNR of each intended user. Therefore, each RIS constitutes a source of interference for the non-intended user. We introduce an analytical approach for characterizing the distribution of the interference-to-noise-ratio (INR) at each receiver, and to study its scaling laws as a function of $N$ in terms of mean, variance, and amount of fading (AF) [8]. Numerical results are illustrated to validate the analysis.

Notation: The main notation is defined in Table I.

\section{SYSTEM MODEL}

We consider the RIS-aided communication system depicted in Fig. 1, which is made of two base stations (BSs), two users (UEs), and two RISs. BS $i$ communicates with $\mathrm{UE}_{i}$ via RIS , $_{i}$ $i=1,2$, where $\mathrm{BS}_{i}$ is equipped with $N_{T}$ transmit antennas, $\mathrm{UE}_{i}$ is equipped with $N_{R}$ receive antennas, and RIS ${ }_{i}$ is made of $N$ reconfigurable elements that apply independent phase shifts to the incident signal. We assume that perfect channel 


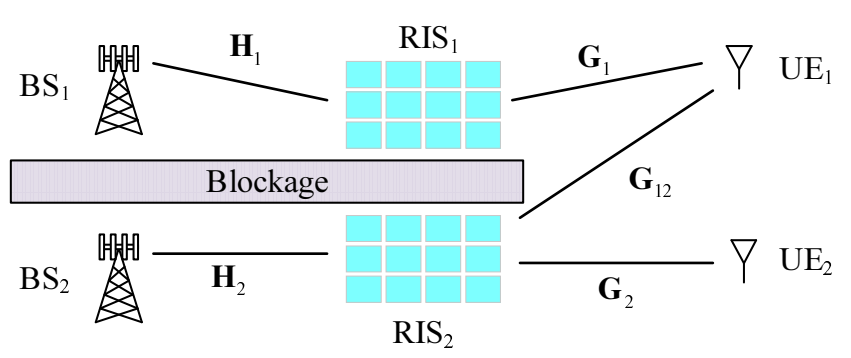

Fig. 1: RIS-aided MIMO communication system.

state information (CSI) is known at the BSs, the UEs, and the RISs. The impact of channel estimation and configuration can be taken into account as recently described in [7], but it is here not considered for simplicity.

The $N_{T} \times 1$ unit-norm beamforming vector at $\mathrm{BS}_{i}$ is denoted by $\mathbf{q}_{i}$ and the $N_{R} \times 1$ unit-norm combining vector at the receiver is denoted by $\mathbf{w}_{i}$. The phase shift applied by the $n$th element of RIS $_{i}$ is denoted as $\phi_{i, n}$. For ease of notation, the $N$ phase shifts of RIS ${ }_{i}$ are collected in the $N \times$ $N$ diagonal matrix $\boldsymbol{\Phi}_{i}, i=1,2$, that is defined as $\boldsymbol{\Phi}_{i}=$ $\operatorname{diag}\left(e^{j \phi_{i, 1}}, e^{j \phi_{i, 2}}, \ldots, e^{j \phi_{i, N}}\right)$. To simplify the analysis, we assume that the channel link from $\mathrm{BS}_{i}$ to $\operatorname{RIS}_{j}(i \neq j)$ is blocked. This implies that the interference is available only at the UEs. Our goal is to study the interference at $\mathrm{UE}_{1}$ caused by $\mathrm{RIS}_{2}$ (a similar analysis can be applied at $\mathrm{UE}_{2}$ ).

Assuming a unit noise power and normalizing with respect to the path-loss, the received signal-to-noise-ratio (SNR) at $\mathrm{UE}_{i}$ can be written as follows [7]:

$$
\mathrm{SNR}_{i}=\left|\mathbf{w}_{i}^{H} \mathbf{G}_{i} \boldsymbol{\Phi}_{i} \mathbf{H}_{i} \mathbf{q}_{i}\right|^{2}
$$

where $\mathbf{H}_{i} \sim \mathcal{C N}\left(\mathbf{0}_{N \times N_{T}}, \mathbf{I}_{N \times N_{T}}\right)$ is the channel from $\mathrm{BS}_{i}$ to RIS R $_{i}$ and $\mathbf{G}_{i} \sim \mathcal{C N}\left(\mathbf{0}_{N_{R} \times N}, \mathbf{I}_{N_{R} \times N}\right)$ is the channel from $\mathrm{RIS}_{i}$ to $\mathrm{UE}_{i}, i=1,2$. Also, $\mathbf{G}_{12} \sim \mathcal{C N}\left(\mathbf{0}_{N_{R} \times N}, \mathbf{I}_{N_{R} \times N}\right)$ is the channel link from RIS $\mathrm{S}_{2}$ to $\mathrm{UE}_{1}$.

The receiver combining vector at $\mathrm{UE}_{i}$, the transmit beamforming vector at $\mathrm{BS}_{i}$, and the phase shifts matrix at $\mathrm{RIS}_{i}$ are jointly optimized to maximize $\mathrm{SNR}_{i}$ in (1). In [7], the authors have recently proved that the maximum $\mathrm{SNR}_{i}$ that is obtained by optimizing the triplet $\left(\mathbf{q}_{i}, \boldsymbol{\Phi}_{i}, \mathbf{w}_{i}\right)$ can be formulated as:

$$
\begin{aligned}
& \mathrm{SNR}_{i} \approx \gamma_{0} N^{2} \max _{l, k}\left\{\lambda_{k, \mathbf{G}_{i}} \lambda_{l, \mathbf{H}_{i}}\left|\Upsilon_{l, k}\right|^{2}\right\} \\
& \Upsilon_{l, k}=\sum_{n=1}^{N}\left|\mathbf{v}_{k, \mathbf{G}}(n)\right|\left|\mathbf{u}_{l, \mathbf{H}}(n)\right|
\end{aligned}
$$

where: (i) $\lambda_{k, \mathbf{G}_{i}}$ is the $k$ th non-zero eigenvalue of the matrix $\mathbf{W}_{\mathbf{G}_{i}}=\frac{1}{N} \mathbf{G}_{i}^{H} \mathbf{G}_{i}$ and $\lambda_{l, \mathbf{H}_{i}}$ is the $l$ th non-zero eigenvalue of the matrix $\mathbf{W}_{\mathbf{H}_{i}}=\frac{1}{N} \mathbf{H}_{i}^{H} \mathbf{H}_{i}$; (ii) $\mathbf{v}_{k, \mathbf{G}_{i}}$ and $\mathbf{u}_{k, \mathbf{G}_{i}}$ are the $k$ th eigenvectors of $\mathbf{W}_{\mathbf{G}_{i}}$ and $\mathbf{W}_{\mathbf{G}_{i}^{H}}$; and (iii) $\mathbf{v}_{l, \mathbf{H}_{i}}$ and $\mathbf{u}_{l, \mathbf{H}_{i}}$ are the $l$ th eigenvectors of $\mathbf{W}_{\mathbf{H}_{i}}$ and $\mathbf{W}_{\mathbf{H}_{i}^{H}}$, respectively.

Denoting the optimal indices $(k, l)$ that maximize $\mathrm{SNR}_{i}$ in (2) by $\left(k_{i}, l_{i}\right)$, the triplet $\left(\mathbf{q}_{i}, \boldsymbol{\Phi}_{i}, \mathbf{w}_{i}\right)$ that maximizes $\mathrm{SNR}_{i}$ is:

$$
\begin{aligned}
\mathbf{w}_{i}^{H} & =\mathbf{u}_{k_{i}, \mathbf{G}_{i}}^{H}, \quad \mathbf{q}_{i}=\mathbf{v}_{l_{i}, \mathbf{H}_{i}} \\
\phi_{i, n} & =-\arg \left\{\mathbf{v}_{k_{i}, \mathbf{G}_{i}}^{*}(n) \mathbf{u}_{l_{i}, \mathbf{H}_{i}}(n)\right\}
\end{aligned}
$$

The distribution of $\mathrm{SNR}_{i}$ in (2) is characterized in [6]. In this paper, on the other hand, we focus our attention on the INR at $\mathrm{UE}_{1}$ that is obtained by considering the optimized triplet $\left(\mathbf{q}_{2}, \mathbf{\Phi}_{2}, \mathbf{w}_{1}\right)$ in $(3)$. In particular, the INR at $\mathrm{UE}_{1}$ can be written as follows:

$$
\mathrm{INR}=\left|\mathbf{w}_{1}^{H} \mathbf{G}_{12} \boldsymbol{\Phi}_{2} \mathbf{H}_{2} \mathbf{q}_{2}\right|^{2}
$$

Let $\mathbf{H}_{i}=\sum_{r=1}^{r_{\mathbf{H}_{i}}} \sqrt{N \lambda_{r, \mathbf{H}_{i}}} \mathbf{u}_{r, \mathbf{H}_{i}} \mathbf{v}_{r, \mathbf{H}_{i}}^{H}$ be the singular value decomposition of $\mathbf{H}_{i}$ where $r_{\mathbf{H}_{i}}=\operatorname{rank}\left(\mathbf{H}_{i}\right)$ is the rank of $\mathbf{H}_{i}$. From (3), we have $\mathbf{H}_{i} \mathbf{q}_{i}=\sqrt{N \lambda_{l_{i}, \mathbf{H}_{i}}} \mathbf{u}_{l_{i}, \mathbf{H}_{i}}$, which implies:

$$
\mathrm{INR}=N \lambda_{l_{2}, \mathbf{H}_{2}}\left|\mathbf{u}_{k_{1}, \mathbf{G}_{1}}^{H} \mathbf{G}_{12} \boldsymbol{\Phi}_{2} \mathbf{u}_{l_{2}, \mathbf{H}_{2}}\right|^{2}
$$

By denoting $\kappa=\mathbf{u}_{k_{1}, \mathbf{G}_{1}}^{H} \mathbf{G}_{12} \boldsymbol{\Phi}_{2} \mathbf{u}_{l_{2}, \mathbf{H}_{2}},(5)$ can be rewritten as follows:

$$
\mathrm{INR}=N \lambda_{l_{2}, \mathbf{H}_{2}}|\kappa|^{2}
$$

Based on [6, Lemma 2], $\mathbf{u}_{k_{1}, \mathbf{G}_{1}}^{H}, \mathbf{u}_{l_{2}, \mathbf{H}_{2}}, \mathbf{v}_{k_{2}, \mathbf{G}_{2}}$ are equivalent in distribution to the following random variables:

$$
\begin{gathered}
\mathbf{u}_{k_{1}, \mathbf{G}_{1}}^{H} \stackrel{d}{=} \hat{\mathbf{u}}_{1}=\mathbf{u}_{1} /\left\|\mathbf{u}_{1}\right\| \\
\mathbf{u}_{l_{2}, \mathbf{H}_{2}} \stackrel{d}{=} \hat{\mathbf{u}}_{2}=\mathbf{u}_{2} /\left\|\mathbf{u}_{2}\right\| \\
\mathbf{v}_{k_{2}, \mathbf{G}_{2}} \stackrel{d}{=} \hat{\mathbf{v}}_{2}=\mathbf{v}_{2} /\left\|\mathbf{v}_{2}\right\|
\end{gathered}
$$

where $\mathbf{u}_{1} \sim \mathcal{C N}\left(\mathbf{0}_{1 \times N_{R}}, \mathbf{1}_{1 \times N_{R}}\right), \mathbf{u}_{2} \sim \mathcal{C N}\left(\mathbf{0}_{1 \times N}, \mathbf{1}_{1 \times N}\right)$, and $\mathbf{v}_{2} \sim \mathcal{C N}\left(\mathbf{0}_{1 \times N}, \mathbf{1}_{1 \times N}\right)$ are mutually independent.

By denoting $\mathbf{p}=\hat{\mathbf{u}}_{1} \mathbf{G}_{12}$, we have $\kappa=\mathbf{p} \boldsymbol{\Phi}_{2} \hat{\mathbf{u}}_{2}$. From the definition of $\boldsymbol{\Phi}_{2}$, we have $\kappa=\sum_{n=1}^{N} \mathbf{p}(n) \hat{\mathbf{u}}_{2}(n) \exp \left(j \phi_{2, n}\right)$. Therefore, $\kappa$ simplifies to:

$$
\kappa=\sum_{n=1}^{N}|\mathbf{p}(n)|\left|\hat{\mathbf{u}}_{2}(n)\right| \exp (j \delta(n))
$$

where, from (3), we have:

$$
\begin{aligned}
\delta(n) & =\arg \left(\mathbf{p}(n) \hat{\mathbf{u}}_{2}(n)\right)-\arg \left\{\mathbf{v}_{k_{2}, \mathbf{G}_{2}}^{*}(n) \hat{\mathbf{u}}_{2}(n)\right\} \\
& =\arg (\mathbf{p}(n))+\arg \left(\mathbf{v}_{k_{2}, \mathbf{G}_{2}}(n)\right)
\end{aligned}
$$

Since $\arg \left(\mathbf{v}_{k_{2}, \mathbf{G}_{2}}(n)\right) \stackrel{d}{=} \arg \left(\mathbf{u}_{l_{2}, \mathbf{H}_{2}}(n)\right) \stackrel{d}{=} \arg \left(\hat{\mathbf{u}}_{2}(n)\right)$, then $\kappa$ in $(8)$ is equivalent in distribution to the following:

$$
\kappa \stackrel{d}{=} \sum_{n=1}^{N} \hat{\mathbf{u}}_{2}(n) \mathbf{p}(n)
$$

Based on (10), we evince that the phase shift $\phi_{2, n}$ introduced by RIS $_{2}$ does not change the distribution of $\kappa$. From (6), the INR can be formulated as follows:

$$
\operatorname{INR} \stackrel{d}{=} N \lambda_{l_{2}, \mathbf{H}_{2}}\left|\sum_{n=1}^{N} \hat{\mathbf{u}}_{2}(n) \mathbf{p}(n)\right|^{2}
$$

Next, we characterize the distribution of the INR in (11).

\section{ANALYSIS OF THE INTERFERENCE-TO-NOISE-RATIO}

In this section, we analyze the distribution, the mean, the variance, and the AF of the INR in [11, as well as the corresponding scaling laws as a function of $N$. 
Theorem 1. Consider the INR in 11. The following approximate equivalent in distribution representation holds true:

$$
\operatorname{INR} \stackrel{d}{\approx} N \lambda_{\mathbf{H}}^{+}\left|\sum_{n=1}^{N} \hat{\mathbf{u}}_{2}(n) \mathbf{p}(n)\right|^{2}
$$

where $\hat{\mathbf{u}}_{1}=\frac{\mathbf{u}_{1}}{\left\|\mathbf{u}_{1}\right\|}, \hat{\mathbf{u}}_{2}=\frac{\mathbf{u}_{2}}{\left\|\mathbf{u}_{2}\right\|}, \mathbf{u}_{1} \sim \mathcal{C N}\left(\mathbf{0}_{1 \times N_{R}}, \mathbf{1}_{1 \times N_{R}}\right)$, $\mathbf{u}_{2} \sim \mathcal{C N}\left(\mathbf{0}_{1 \times N}, \mathbf{1}_{1 \times N}\right), \mathbf{p}(n)=\sum_{m=1}^{N_{R}} \hat{\mathbf{u}}_{1}(m) \mathbf{G}_{12}(m, n)$, $\mathbf{G}_{12} \sim \mathcal{C N}\left(\mathbf{0}_{N_{R} \times N}, \mathbf{1}_{N_{R} \times N}\right)$, and $\lambda_{\mathbf{H}}^{+}$is a Gamma $R V$, whose mean and variance are defined as follows:

$$
\begin{aligned}
& \mathbb{E}\left(\lambda_{\mathbf{H}}^{+}\right)=\alpha_{1}\left(N_{T}, N\right)-\alpha_{0} \beta_{1}\left(N_{T}, N\right) \\
& \mathbb{V}\left(\lambda_{\mathbf{H}}^{+}\right)=\beta_{0} \beta_{1}^{2}\left(N_{T}, N\right)
\end{aligned}
$$

where $\alpha_{0}=1.7711, \beta_{0}=0.8132$ and:

$$
\begin{aligned}
& \alpha_{1}\left(N_{T}, N\right)=\left(1+\sqrt{N_{T} / N}\right)^{2} \\
& \beta_{1}\left(N_{T}, N\right)=N^{-2 / 3}\left(1+\sqrt{N_{T} / N}\right)\left(1+\sqrt{N / N_{T}}\right)^{1 / 3}
\end{aligned}
$$

Proof. From [6, Lemma 3], the eigenvectors and the eigenvalues of a Wishart matrix with zero mean complex Gaussian entries are independent. Also, $\lambda_{l_{2}, \mathbf{H}_{2}}$ in (11) is the maximum eigenvalue of the $\mathbf{W}_{\mathbf{H}_{2}}=\frac{1}{N} \mathbf{H}_{2}^{H} \mathbf{H}_{2}$ whose distribution can be well approximated with a Gamma RV as stated in [6, Lemma 1] based on [9, 10]. This concludes the proof.

Theorem 2. If $N \gg 1$, the following holds true:

$$
\mathrm{INR}^{N \gg 1} \frac{N}{2} \lambda_{\mathbf{H}}^{+} Q
$$

where $\lambda_{\mathbf{H}}^{+} \sim \mathcal{G}\left(\left(\mathbb{E}\left(\lambda_{\mathbf{H}}^{+}\right)\right)^{2} / \mathbb{V}\left(\lambda_{\mathbf{H}}^{+}\right), \mathbb{V}\left(\lambda_{\mathbf{H}}^{+}\right) / \mathbb{E}\left(\lambda_{\mathbf{H}}^{+}\right)\right)$is a Gamma $R V$ with $\mathbb{E}\left(\lambda_{\mathbf{H}}^{+}\right)$and $\mathbb{V}\left(\lambda_{\mathbf{H}}^{+}\right)$given in (13), and $Q \sim \chi^{2}(2)$ is a Chi-Squared $R V$ with two degrees of freedom.

Proof. From Theorem 1, $\lambda_{\mathbf{H}}^{+}$can be approximated as a Gamma random variable as follows:

$$
\lambda_{\mathbf{H}}^{+} \sim \mathcal{G}\left(\left(\mathbb{E}\left(\lambda_{\mathbf{H}}^{+}\right)\right)^{2} / \mathbb{V}\left(\lambda_{\mathbf{H}}^{+}\right), \mathbb{V}\left(\lambda_{\mathbf{H}}^{+}\right) / \mathbb{E}\left(\lambda_{\mathbf{H}}^{+}\right)\right)
$$

For $N \gg 1$, by virtue of the central limit theorem, $\kappa=\sum_{n=1}^{N} \hat{\mathbf{u}}_{2}(n) \mathbf{p}(n)$ in (12) can be approximated with a Gaussian RV. By definition, $\mathbf{p}=\hat{\mathbf{u}}_{1} \mathbf{G}_{12}$, and by denoting $\vartheta(n, m)=\hat{\mathbf{u}}_{1}(m) \hat{\mathbf{u}}_{2}(n) \mathbf{G}_{12}(m, n)$, we have:

$$
\begin{aligned}
\mathbb{E}(\kappa) & =\mathbb{E}\left(\sum_{n=1}^{N} \hat{\mathbf{u}}_{2}(n) \mathbf{p}(n)\right) \\
& =\sum_{n=1}^{N} \sum_{m=1}^{N_{R}} \mathbb{E}(\vartheta(n, m))
\end{aligned}
$$

Since $\mathbf{G}_{12}, \hat{\mathbf{u}}_{1}$ and $\hat{\mathbf{u}}_{2}$ are mutually independent and $\mathbb{E}\left(\mathbf{G}_{12}(m, n)\right)=0$, we have $\mathbb{E}(\vartheta(n, m))=0$ and $\mathbb{E}(\kappa)=0$. Thus, $\mathbb{V}(\kappa)=\mathbb{E}\left((\kappa-\mathbb{E}(\kappa))(\kappa-\mathbb{E}(\kappa))^{*}\right)=\mathbb{E}\left(\kappa \kappa^{*}\right)$ is:

$$
\mathbb{V}(\kappa)=\mathbb{E}\left(\sum_{n_{1}=1}^{N} \sum_{m_{1}=1}^{N_{R}} \sum_{n_{2}=1}^{N} \sum_{m_{2}=1}^{N_{R}} \vartheta\left(n_{1}, m_{1}\right) \vartheta^{*}\left(n_{2}, m_{2}\right)\right)
$$

If $\left(n_{1}, m_{1}\right) \neq\left(n_{2}, m_{2}\right), \mathbf{G}_{12}\left(m_{1}, n_{1}\right)$ and $\mathbf{G}_{12}\left(m_{2}, n_{2}\right)$ are independent, then $\mathbb{E}\left(\mathbf{G}_{12}\left(m_{1}, n_{1}\right) \mathbf{G}_{12}^{*}\left(m_{2}, n_{2}\right)\right)=$ $\mathbb{E}\left(\mathbf{G}_{12}\left(m_{1}, n_{1}\right)\right) \mathbb{E}\left(\mathbf{G}_{12}^{*}\left(m_{2}, n_{2}\right)\right)=0$. Therefore, (18) is non-zero only for $\left(n_{1}, m_{1}\right)=\left(n_{2}, m_{2}\right)$, and it simplifies to:

$$
\mathbb{V}(\kappa)=\sum_{n_{1}=1}^{N} \sum_{m_{1}=1}^{N_{R}} \mathbb{E}\left(\left|\vartheta\left(n_{1}, m_{1}\right)\right|^{2}\right)
$$

From [6, Lemma 4], we obtain $\mathbb{E}\left(\left|\hat{\mathbf{u}}_{1}\left(m_{1}\right)\right|^{2}\right)=\frac{1}{N_{R}}$, $\mathbb{E}\left(\left|\hat{\mathbf{u}}_{\mathbf{2}}\left(n_{1}\right)\right|^{2}\right)=\frac{1}{N}$. Also, $\mathbb{E}\left(\left|\mathbf{G}_{12}\left(m_{1}, n_{1}\right)\right|^{2}\right)=1$ because $\mathbf{G}_{12}\left(m_{1}, n_{1}\right) \sim \mathcal{C N}(0,1)$. Therefore, 19$)$ is equal to:

$$
\mathbb{V}(\kappa)=1
$$

From the central limit theorem, we have $\kappa \sim \mathcal{C N}(0,1)$. Consequently, $\sqrt{2} \Re(\kappa), \sqrt{2} \Im(\kappa) \sim \mathcal{N}(0,1)$ and $Q=2|\kappa|^{2}$ can be written as $Q=(\sqrt{2} \Re(\kappa))^{2}+(\sqrt{2} \Im(\kappa))^{2} \sim \chi^{2}(2)$. This concludes the proof.

Based on Theorem 2, we evince that, for $N \gg 1$, the INR is the product of an (approximate) Gamma RV and a ChiSquare RV with two degrees of freedom. Also, the two RVs are mutually independent. In the following propositions, we characterize the mean, the variance, and the AF of the INR based on Theorem 2 .

Proposition 1. Assume $N \gg 1$. The mean and the variance of the INR in (15) can be formulated as follows:

$$
\begin{aligned}
& \mathbb{E}(\mathrm{INR}) \stackrel{N \geqq 1}{=} N \mathbb{E}\left(\lambda_{\mathbf{H}}^{+}\right) \\
& \mathbb{V}(\mathrm{INR}) \stackrel{N \gg 1}{=} 2 N^{2} \mathbb{V}\left(\lambda_{\mathbf{H}}^{+}\right)+N^{2}\left(\mathbb{E}\left(\lambda_{\mathbf{H}}^{+}\right)\right)^{2}
\end{aligned}
$$

Proof. From [15], we obtain:

$$
\begin{aligned}
& \mathbb{E}(\mathrm{INR}) \stackrel{N \gg 1}{=}(N / 2) \mathbb{E}\left(\lambda_{\mathbf{H}}^{+}\right) \mathbb{E}(Q) \stackrel{(a)}{=} N \mathbb{E}\left(\lambda_{\mathbf{H}}^{+}\right) \\
& \mathbb{E}\left(\mathrm{INR}^{2}\right) \stackrel{N \gg 1}{=}(N / 2)^{2} \mathbb{E}\left(\left(\lambda_{\mathbf{H}}^{+}\right)^{2}\right) \mathbb{E}\left(Q^{2}\right) \\
& \stackrel{(b)}{=} 2 N^{2} \mathbb{E}\left(\left(\lambda_{\mathbf{H}}^{+}\right)^{2}\right)
\end{aligned}
$$

where $(a)$ follows from $\mathbb{E}(Q)=2$ and $(b)$ follows from $\mathbb{E}\left(Q^{2}\right)=\mathbb{V}(Q)+(\mathbb{E}(Q))^{2}=8$. Using $\mathbb{E}\left(\left(\lambda_{\mathbf{H}}^{+}\right)^{2}\right)=\mathbb{V}\left(\lambda_{\mathbf{H}}^{+}\right)+$ $\left(\mathbb{E}\left(\lambda_{\mathbf{H}}^{+}\right)\right)^{2}$ and $\mathbb{V}(\mathrm{INR})=\mathbb{E}\left(\mathrm{INR}^{2}\right)-(\mathbb{E}(\mathrm{INR}))^{2}$, we obtain (21). This concludes the proof.

From Proposition 1 , we can infer the scaling laws of the mean, variance, and $\mathrm{AF}$ as a function of $N$ by taking into account the distribution of $\lambda_{\mathbf{H}}^{+}$in Theorem 1. The final result is formally stated in the following proposition.

Proposition 2. Assuming $N \gg 1$ and let the $A F$ of the INR be $\mathrm{AF}_{\mathrm{INR}}=\mathbb{V}(\mathrm{INR}) /(\mathbb{E}(\mathrm{INR}))^{2}$. As a function of $N$, while keeping the other parameters fixed, the following scaling laws hold:

$$
\begin{aligned}
& \mathbb{E}(\mathrm{INR}) \stackrel{N \gg 1}{\propto} N \\
& \mathbb{V}(\mathrm{INR}) \stackrel{N \gg 1}{{ }^{2}} N^{2} \\
& \mathrm{AF}_{\mathrm{INR}} \stackrel{N \gg 1}{\propto} 1
\end{aligned}
$$



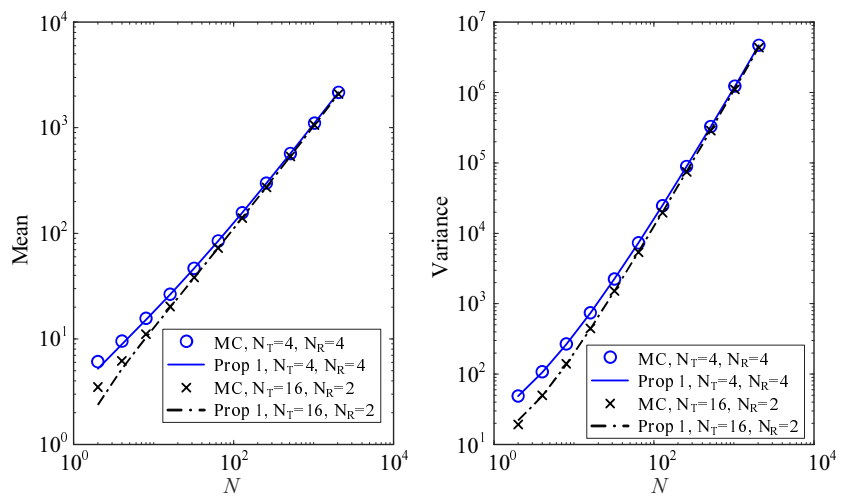

Fig. 2: Mean and variance of the INR.
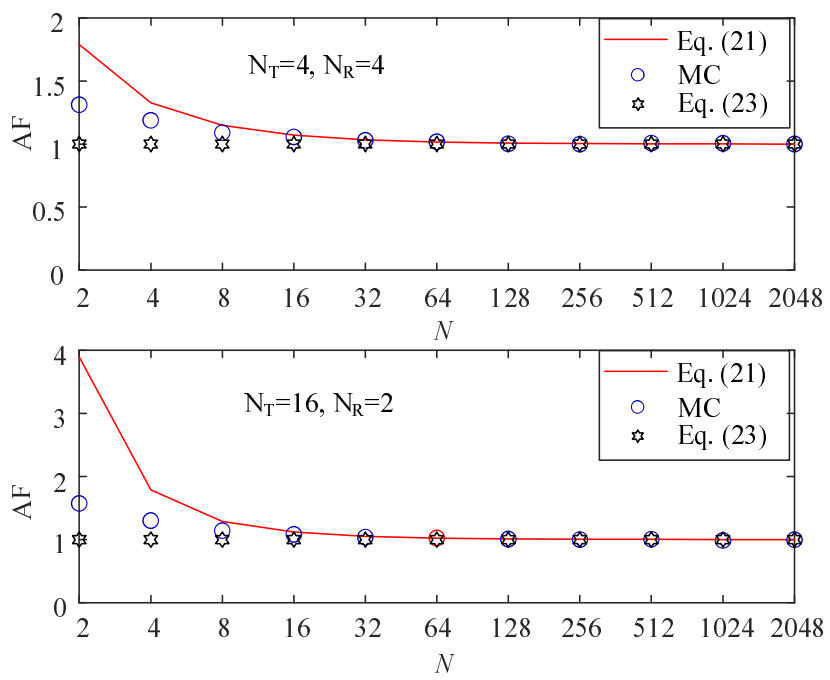

Fig. 3: AF of the INR.

Proof. Consider the definitions in (12) and (13). When $N \gg 1$, we have $\left(1+\sqrt{N_{T} / N}\right) \stackrel{N \gg 1}{\propto 1} 1$ and $\left(1+\sqrt{N / N_{T}}\right)$ $N \gg 1 \sqrt{N / N_{T}}$. Thus, we obtain $\alpha_{1}\left(N_{T}, N\right) \stackrel{N \gg 1}{\propto} 1$ and $\beta_{1}\left(N_{T}, N\right) \stackrel{N \gg 1}{\propto} N_{T}^{-1 / 6} N^{-1 / 2}$, and, from [13), we have:

$$
\begin{gathered}
\mathbb{E}\left(\lambda_{\mathbf{H}}^{+}\right)=\alpha_{1}\left(N_{T}, N\right)-\alpha_{0} \beta_{1}\left(N_{T}, N\right) \\
{ } \gg 1 \\
\stackrel{1}{ } 1-\alpha_{0} N_{T}{ }^{-1 / 6} N^{-1 / 2}{ }^{N \gg 1} 1 \\
\mathbb{V}\left(\lambda_{\mathbf{H}}^{+}\right)=\beta_{0} \beta_{1}^{2}\left(N_{T}, N\right) \stackrel{N \gg 1}{\propto} \beta_{0} N_{T}{ }^{-1 / 3} N^{-1}
\end{gathered}
$$

Therefore, from 21], we obtain:

$$
\begin{aligned}
& \mathbb{E}(\mathrm{INR}) \stackrel{N \gg 1}{=} N \mathbb{E}\left(\lambda_{\mathbf{H}}^{+}\right) \stackrel{N \gg 1}{\propto} N \\
& \mathbb{V}(\mathrm{INR}) \stackrel{N \geqq 1}{=} 2 N^{2} \mathbb{V}\left(\lambda_{\mathbf{H}}^{+}\right)+N^{2}\left(\mathbb{E}\left(\lambda_{\mathbf{H}}^{+}\right)\right)^{2} \\
& \stackrel{N \gg 1}{\propto} 2 \beta_{0} N_{T}^{-1 / 3} N+N^{2} \stackrel{N \gg 1}{\propto} N^{2}
\end{aligned}
$$

Finally, we have $\mathrm{AF}_{\text {INR }}=\mathbb{V}(\mathrm{INR}) /(\mathbb{E}(\mathrm{INR}))^{2} \stackrel{N \gg 1}{\propto} 1$. This concludes the proof.

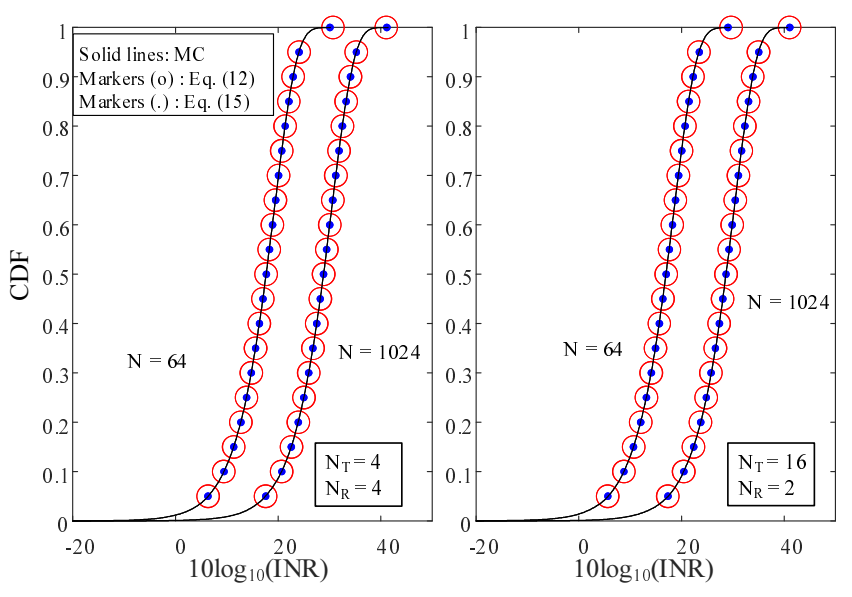

Fig. 4: CDF of the INR: Simulations vs. Theorems 1 and 2.

\section{NUMERICAL RESULTS}

In this section, we illustrate some numerical results in order to substantiate the obtained analytical findings against Monte Carlo (MC) simulations. Figure 2 shows the mean and variance of the INR, and compares MC simulations and Proposition 1. Figure 3 compares the AF obtained from MC simulations against the analytical definition of the $\mathrm{AF}$ obtained from 21), and the scaling laws in 23). In both figures, we obtain a good agreement for $N \gg 1$. Finally, Fig. 4 reports the cumulative distribution function (CDF) of the INR obtained from $\mathrm{MC}$ simulations, and compares it against the corresponding CDF obtained from Theorems 1 and 2. Also in this case, we obtain a good agreement between analysis and simulations.

By comparing the distribution of the INR with that of the SNR obtained in [6], we evince that an RIS is capable of reducing the $\mathrm{AF}$ of the intended link linearly with $N$, while this scaling law is not observed for the INR (i.e., the AF is constant with $N$ ). The study conducted in this paper highlights that the interference-aware optimization of RISs may be needed to achieve a better robustness to the interference.

\section{CONCLUSION}

We have introduced an analytical framework to characterize the distribution of the INR in RIS-aided systems. Over Rayleigh fading, in particular, we have proved that the INR is asymptotically equivalent in distribution to the product of a Gamma RV and a Chi-Square RV. Also, the AF is proved to be independent of the number $N$ of reconfigurable elements.

Generalizations of the results reported in this paper include the analysis and optimization of RIS-assisted communications based on electromagnetic-compliant communication models, such as that recently introduced in [11] and [12].

\section{ACKNOWLEDGEMENT}

This work was supported in part by the European Commission through the H2020 5GStepFWD project under grant agreement number 722429 and through the H2020 ARIADNE project under grant agreement number 871464 . 


\section{REFERENCES}

[1] M. Di Renzo et al., "Smart radio environments empowered by reconfigurable AI meta-surfaces: An idea whose time has come," EURASIP Journal on Wireless Communications and Networking, vol. 2019, no. 1, pp. 1-20, 2019.

[2] M. Di Renzo et al., "Smart radio environments empowered by reconfigurable intelligent surfaces: How it works, state of research, and the road ahead," IEEE Journal on Selected Areas in Communications, vol. 38, pp. 2450-2525, 2020.

[3] W. Tang et al., "Wireless communications with reconfigurable intelligent surface: Path loss modeling and experimental measurement," IEEE Transactions on Wireless Communications, vol. 20, no. 1, pp. 421-439, 2021.

[4] F. H. Danufane et al., "On the path-loss of reconfigurable intelligent surfaces: An approach based on green's theorem applied to vector fields," arXiv preprint arXiv:2007.13158, 2020.

[5] M. Di Renzo et al., "Reconfigurable intelligent surfaces vs. relaying: Differences, similarities, and performance comparison," IEEE Open Journal of the Communications Society, vol. 1, pp. 798-807, 2020.

[6] X. Qian et al., "Beamforming through reconfigurable intelligent surfaces in single-user mimo systems: Snr distribution and scaling laws in the presence of channel fading and phase noise," IEEE Wireless Communications Letters, vol. 10, no. 1, pp. 77-81, 2021.

[7] A. Zappone et al., "Overhead-aware design of reconfigurable intelligent surfaces in smart radio environments," IEEE Transactions on Wireless Communications, vol. 20, no. 1, pp. 126-141, 2021.

[8] B. Holter and G. E. Oien, "On the amount of fading in mimo diversity systems," IEEE Transactions on Wireless Communications, vol. 4, no. 5, pp. 2498-2507, 2005.

[9] M. Chiani, "Distribution of the largest eigenvalue for real wishart and gaussian random matrices and a simple approximation for the tracy-widom distribution," Journal of Multivariate Analysis, vol. 129, pp. 69-81, 2014.

[10] L. Wei and O. Tirkkonen, "Analysis of scaled largest eigenvalue based detection for spectrum sensing," in 2011 IEEE International Conference on Communications (ICC). IEEE, 2011, pp. 1-5.

[11] G. Gradoni and M. Di Renzo, "End-to-end mutualcoupling-aware communication model for reconfigurable intelligent surfaces: An electromagneticcompliant approach based on mutual impedances,"
IEEE Wireless Communications Letters, IEEE Early Access, 2021.

[12] X. Qian and M. Di Renzo, "Mutual coupling and unit cell aware optimization for reconfigurable intelligent surfaces," arXiv preprint arXiv:2011.14373, 2021. 